\title{
Mechanism of Arsenic Monolayer Doping of oxide-free Si(111)
}

R. C. Longo, ${ }^{1 \dagger}$ E. C. Mattson,,${ }^{1 \dagger}$ A. Vega, ${ }^{1 \dagger}$ W. Cabrera,${ }^{1} \mathrm{~K}$. Cho, ${ }^{1}$ Y. J. Chabal, ${ }^{1}$ and P. Thissen $^{2}$

${ }^{1}$ Department of Materials Science \& Engineering, The University of Texas at Dallas, Richardson, Texas 75080, USA

${ }^{2}$ Karlsruher Institut fur Technologie (KIT), Institut fur Funktionelle Grenzflachen (IFG), Hermann-von-Helmholtz-Platz 1, 76344 Eggenstein-Leopoldshafen, Germany ${ }^{\dagger}$ Equal Contribution 


\begin{tabular}{|c|c|}
\hline Supplemental Note 1 & $\begin{array}{l}\text { Experimental and computational } \\
\text { methods }\end{array}$ \\
\hline Figure S1 & $\begin{array}{l}\text { Temperature-dependent IR Spectra of } \\
\text { MasA-Functionalized Si(100) }\end{array}$ \\
\hline Figure S2 & $\begin{array}{c}\text { Adsorption kinetic energy profile as a } \\
\text { function of the distance between } \\
\text { MAsA and } \mathrm{Si}(111)-\mathrm{H}\end{array}$ \\
\hline Figure S3 & $\begin{array}{c}\text { Temperature-dependent differential } \\
\text { IR transmission spectra of } \\
\text { MasA/Si(111) }\end{array}$ \\
\hline Figure S4 & $\begin{array}{l}\text { LEIS spectra of MasA/Si(111) as- } \\
\text { prepared and as a function of } \\
\text { temperature }\end{array}$ \\
\hline Supplemental Note 2 & Quantitative LEIS Analysis \\
\hline Figure S5 & $\begin{array}{l}\text { LEIS Evaluation of As Depth via TRBS } \\
\text { Modeling }\end{array}$ \\
\hline Figure S6 & $\begin{array}{l}\text { Determination of the reionization } \\
\text { function used for quantitative LEIS } \\
\text { modeling }\end{array}$ \\
\hline Supplemental Note 3 & $\begin{array}{l}\text { Approach for fitting broadband } \\
\text { differential IR spectra }\end{array}$ \\
\hline Figure S17 & $\begin{array}{l}\text { TOF-SIMS Measurements of MAsA ML- } \\
\text { Doped Si(111) }\end{array}$ \\
\hline Figure S8 & $\begin{array}{l}\text { Kinetic Barriers for } \mathrm{CH}_{3} \text { desorption } \\
\text { from } \mathrm{MAsA}\end{array}$ \\
\hline Figure S9 & $\begin{array}{l}\text { Relative energies for different As } \\
\text { doping configurations }\end{array}$ \\
\hline Figure S10 & $\begin{array}{l}\text { Kinetic barrier for As diffusion into } \\
\text { Si(111) }\end{array}$ \\
\hline Figure S11 & $\begin{array}{c}\text { TOF-SIMS Profiles of P in ODPA ML- } \\
\text { Doped Si(111) }\end{array}$ \\
\hline Figure S12 & $\begin{array}{c}\text { Electrical Characterization of ODPA } \\
\text { ML-Doped } \mathrm{Si}(111)\end{array}$ \\
\hline Figure S13 & $\begin{array}{l}\text { Electrical Characteriation of clean } \\
\mathrm{Si}(111)-\mathrm{H}\end{array}$ \\
\hline
\end{tabular}




\section{Supplemental Note 1: Experimental and Computational Methodology}

Sample Preparation

A $3.8 \mathrm{~cm} \times 1.5 \mathrm{~cm}$ wafer of Float Zone (FZ) Si(111), double side polished, was cleaned by the RCA method: the sample is dipped in SC-1 solution $\left(5: 1: 1 \mathrm{H}_{2} \mathrm{O}: \mathrm{H}_{2} \mathrm{O}_{2}: \mathrm{NH}_{4} \mathrm{OH}\right)$ at $80{ }^{\circ} \mathrm{C}$ for 10 minutes, and SC-2 solution $\left(5: 1: 1 \mathrm{H}_{2} \mathrm{O}: \mathrm{H}_{2} \mathrm{O}_{2}: \mathrm{HCl}\right)$ at $80{ }^{\circ} \mathrm{C}$ for $10 \mathrm{~min}$, followed by thorough $\mathrm{DI}$ water (18.2 $\mathrm{M} \Omega \mathrm{cm}$ MiliQ) rinsing and blow-drying with nitrogen $\left(\mathrm{N}_{2}\right)$ gas. The cleaned samples were then $\mathrm{H}$-terminated by a $60 \mathrm{~s}$ dip in $10 \%$ $\mathrm{HF}(\mathrm{aq})$, followed by a 15 min dip in $40 \% \mathrm{NH}_{4} \mathrm{~F}(\mathrm{aq})$, and a final rinse in $\mathrm{H}_{2} \mathrm{O}$ for $30 \mathrm{~s}$. This produces an atomically smooth (111)-oriented Si surface from tens to hundreds of nanometers. Afterward the sample is coated with methylarsenic acid (MAsA) by immersion into a $10^{-3} \mathrm{~mol}$ methanol solution at $65^{\circ} \mathrm{C}$ for $12 \mathrm{~h}$ (in a glovebox). The environment control is critical to avoid oxidation of the surface. In situ heating measurements were performed via direct resistive heating of the Si substrate. The heating rates, although not controlled directly, were approximately $200^{\circ} \mathrm{C} / \mathrm{min}$, and the sample was maintained at the target temperature for approximately $10 \mathrm{~s}$ before cooling.

Fourier Transform Infrared Spectroscopy (FTIR)

IR absorption data was recorded in the dry $\mathrm{N}_{2}(\mathrm{~g})$-purged bench of a Fourier transform infrared (FTIR) spectrometer (Nicolet 6700). Spectra were obtained with a nominal 4 $\mathrm{cm}^{-1}$ resolution between 400 and $4000 \mathrm{~cm}^{-1}$ in transmission, at an angle of incidence of $74^{\circ}$ with respect to the Si surface normal. A room temperature pyroelectric detector (DTGS) was used for data collection. Three consecutive loops, each consisting of 500 single beam spectral scans, were performed for each sample condition.

X-ray Photoelectron Spectroscopy (XPS)

XPS analysis were performed with a Quantum 2000 Scanning ESCA Microprobe (Physical Electronics, USA) spectrometer, equipped with a concentric hemispherical analyzer under ultra-high vacuum (UHV) conditions ( $10^{-9}$ mbar) using an Al Ka1,2radiation source. Spectra were recorded at $45^{\circ}$ take-off angle with respect to the surface. A sample area of $100 \mu \mathrm{m} \times 100 \mu \mathrm{m}$ was analyzed with a pass energy of 46.95 $\mathrm{eV}$ for survey and $11.75 \mathrm{eV}$ for detailed elemental scans. The spectra obtained were analyzed using the CASA XPS software, and the surface sensitivity factors used to determine the atomic concentrations were taken from the MultiPak Version 6.0 software (supplied by Physical Electronics).

Low Energy Ion Scattering (LEIS)

LEIS measurements were done using a Qtac analyzer (ION-TOF GmbH, Münster, Germany), applying a $3.0 \mathrm{keV} 4 \mathrm{He}^{+}$beam at a beam current of $2.3 \mathrm{nA}$ and a fixed scatter angle of $145^{\circ}$. Each measurement lasted 120 seconds. The fraction of backscattered He-ions was measured as a function of the kinetic energy with a double toroidal analyzer, imaging the ions according to their energy onto a position sensitive electrostatic detector. The use of this analyzer-detector combination was important because the large scatter angle of detection combined with the parallel detection 
increases the sensitivity by two orders of magnitude and allows a reduction of the overall ion dose needed for measurements. Consequently, the total ion dose was very low compared to the surface atomic density and, therefore, ion-induced sputtering and intermixing were negligible.

\section{TOF-SIMS}

Time-of-flight Secondary Ion Mass Spectrometry (TOF-SIMS) was performed using two separate experimental setups. In the case of As-MLD using MAsA, an IonTOF TOFSIMS V instrument equipped with a Bi liquid metal ion gun and a Cs sputtering gun. The Cs sputtering was performed over a $140 \times 140 \mu \mathrm{m}^{2}$ area using a $1 \mathrm{keV}, 10 \mathrm{nA}$ beam, and a $25 \mathrm{keV}, 0.4 \mathrm{pA} \mathrm{Bi}$ analysis beam was applied to a $50 \times 50 \mu^{2}$ area at the center of the sputtering area. Three separate regions of the sample were examined for reproducibility. The As concentration was evaluated from an As-implanted Si sample with known As concentration. In the case of P-MLD using octadecylphosphonic acid described below, the measurements were carried out with a gridless reflectron-based ToF-SIMS V (ION-TOF GmbH, Muenster, Germany), equipped with a bismuth-cluster ion source. All spectra and images were obtained using $\mathrm{Bi}^{3+}$ primary ions at $25 \mathrm{keV}$ energy in the high current bunched mode, with a mass resolution of $\mathrm{m} / \Delta \mathrm{m}=6000$. The beam diameter was about $3-5 \mu \mathrm{m}$ and all measurements were made under static conditions (primary ion dose $<\mathrm{FPI}=5 \times 10^{12}$ ions $\mathrm{cm}^{-2}$ ) on an area of $500 \times 500 \mu \mathrm{m}^{2}$ with 256 pixels.

\section{Electrochemical Impedance Spectroscopy}

Impedance spectroscopy of the ODPA-functionalized and annealed samples was performed using an IMPEDANCE ANALYZER IM3570 (HIOKI) R, L, C measuring device operated at room temperature in the frequency range of $5 \mathrm{~Hz}-500 \mathrm{MHz}$, using titanium clamping contacts with the diameter of $1 \mathrm{~mm}$. The measurements were performed in a glovebox.

\section{Computational Methods}

The calculations were performed using density-functional theory (DFT) within the generalized gradient approximation (GGA) as implemented in the Vienna ab initio simulation package (VASP)., ${ }^{1,2}$ The electron-ion interaction was described within the projector-augmented wave (PAW) scheme. $^{3}$ The electronic wave functions were expanded into plane waves up to a kinetic energy of $360 \mathrm{eV}$. The surface was modeled by periodically repeated slabs. The supercell used here consisted of 8 atomic layers of Si plus adsorbed molecules and a vacuum region equivalent to 16 atomic layers. The 7 uppermost layers of Si as well as the adsorbate degrees of freedom were allowed to relax until the forces on the atoms were below $10 \mathrm{meV} / \AA$. The Brillouin zone integration was performed using a $4 \times 4 \times 1$ mesh within the Monkhorst-Pack scheme. ${ }^{4}$ The PBE functional was used to describe the electron exchange and correlation energy within the GGA. ${ }^{5}$ Ab initio Molecular Dynamics (MD) simulations were performed using the velocity Verlet algorithm coupled with the Nose thermostat to solve the equations of motion. Starting at $0 \mathrm{~K}$, the temperature was increased in steps of $50 \mathrm{~K}$. At each temperature, a time step of 0.5 fs was used during an equilibration period of 10000 steps, for a total simulation time of 5 ps. Kinetic barriers were calculated by the 
nudged elastic band (NEB) method, using a string of geometric configurations to describe the reaction pathway of the system. ${ }^{6,7} \mathrm{~A}$ spring interaction between every configuration ensured continuity of the reaction pathway. 


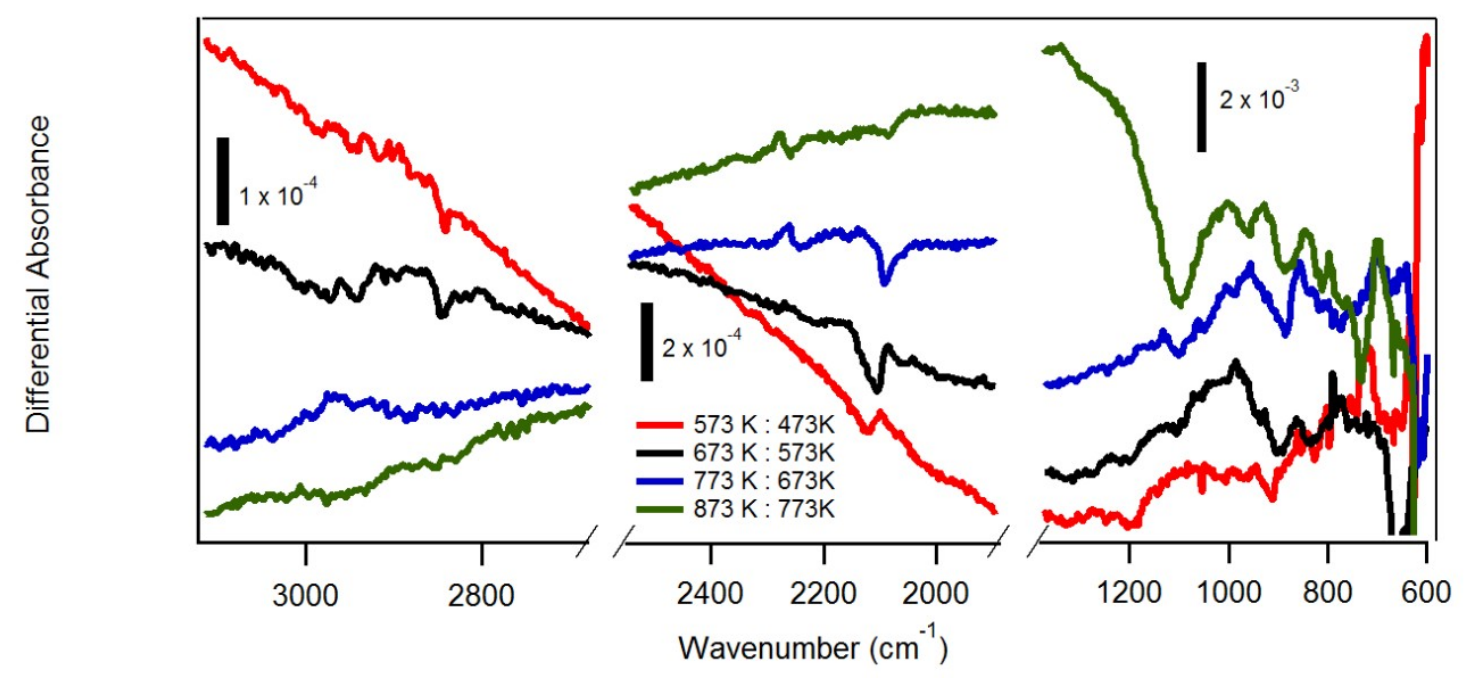

Figure S1. In situ IR Absorbance Measurements of As-MLD of analogously prepared $\mathrm{Si}(100)$ surfaces, shown to highlight the chemical similarity between the MLD process on $\mathrm{Si}(100)$ and $\mathrm{Si} \mathrm{(111)} \mathrm{surfaces.} \mathrm{The} \mathrm{data} \mathrm{show} \mathrm{desorption} \mathrm{of} \mathrm{methyl} \mathrm{ligands} \mathrm{follow-}$ ing annealing at $673 \mathrm{~K}$ in conjunction with the development of a broad band between 1000-1200 $\mathrm{cm}^{-1}$ associated with As-O, as seen on the Si(111) surfaces. Following annealing to 773K, oxygen insertion into Si backbonds is evidenced by the development of bands at 2145,2180 and $2280 \mathrm{~cm}^{-1}$, as well as the development of several modes in the fingerprint region characteristic of substoichiometric oxides. Following annealing to $873 \mathrm{~K}$, the intense negative feature at $1100 \mathrm{~cm}^{-1}$ indicates a loss of $A s=0$ stretch bonds. 


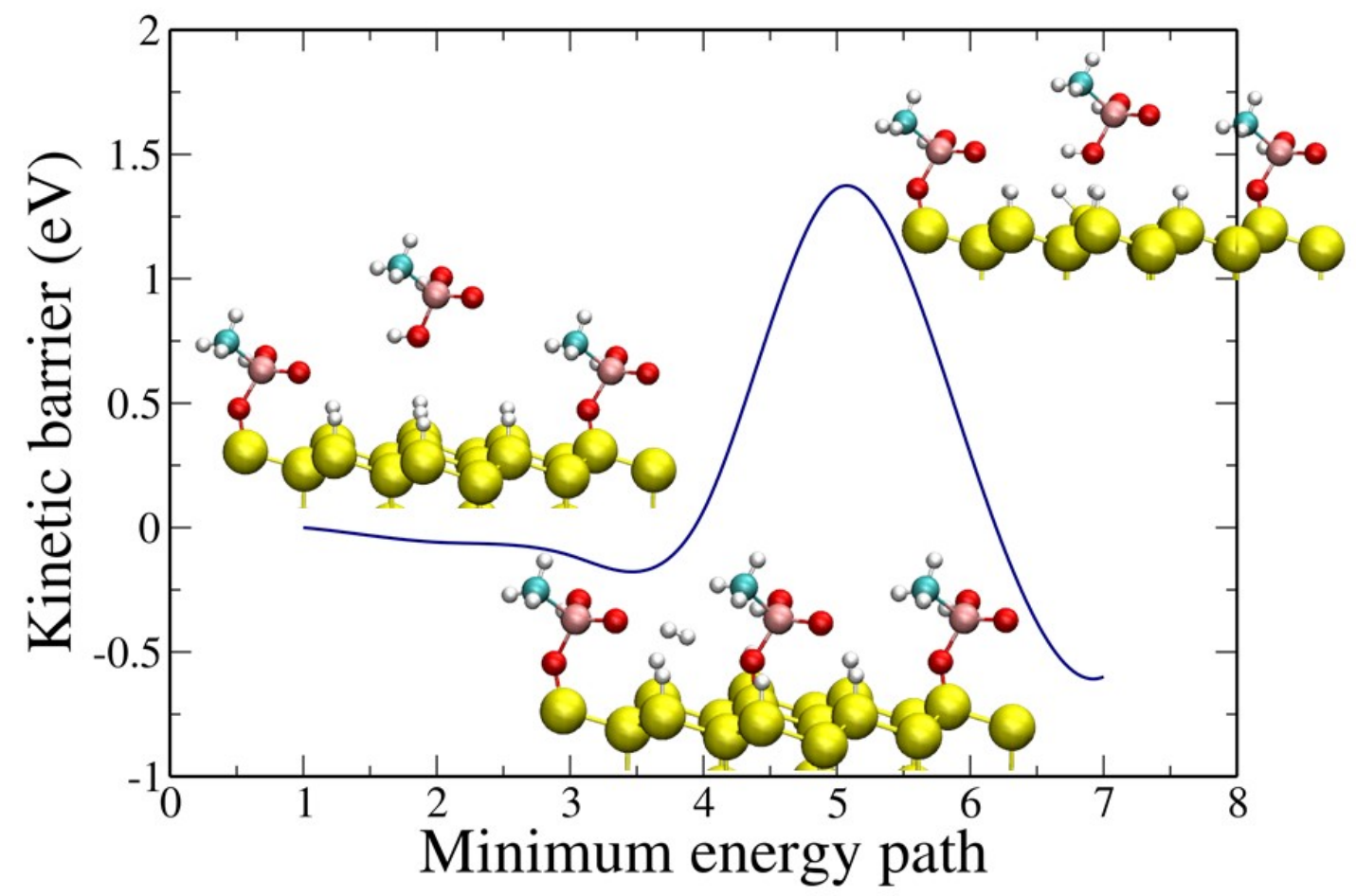

Figure S2. Adsorption kinetic energy profile as a function of the distance between the MAsA molecule and the oxide-free $\mathrm{H}$-terminated $\mathrm{Si}(111)$ surface. Yellow spheres represent $\mathrm{Si}$ atoms; white, $\mathrm{H}$; blue, $\mathrm{C}$, red, $\mathrm{O}$ and pink spheres, As atoms, respectively. 


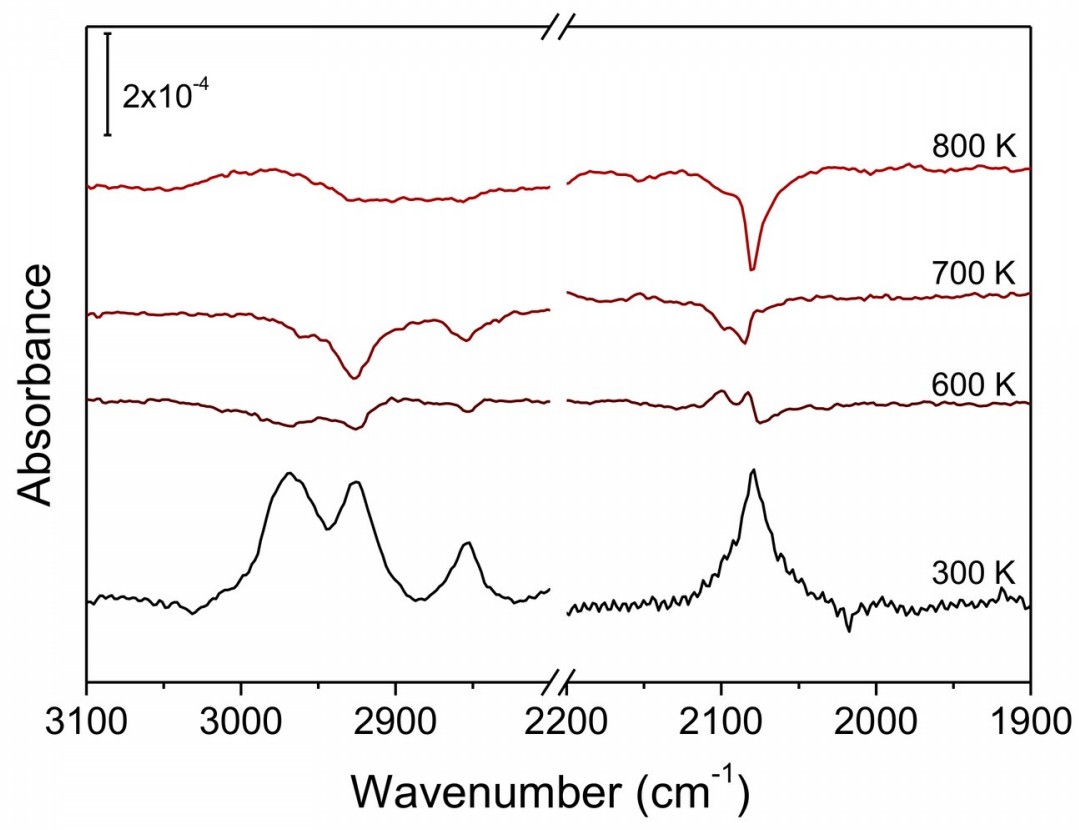

Figure S3. Differential transmission infrared spectra of MAsA grafted on $\mathrm{H}$-terminated $\mathrm{Si}(111)$ as a function of temperature. All spectra are referenced to spectra recorded at the previous lower temperature. The bottom spectrum (black, 300K) is referenced to the piranha-cleaned sample, prior to $\mathrm{H}$ termination and MAsA treatment. 
A

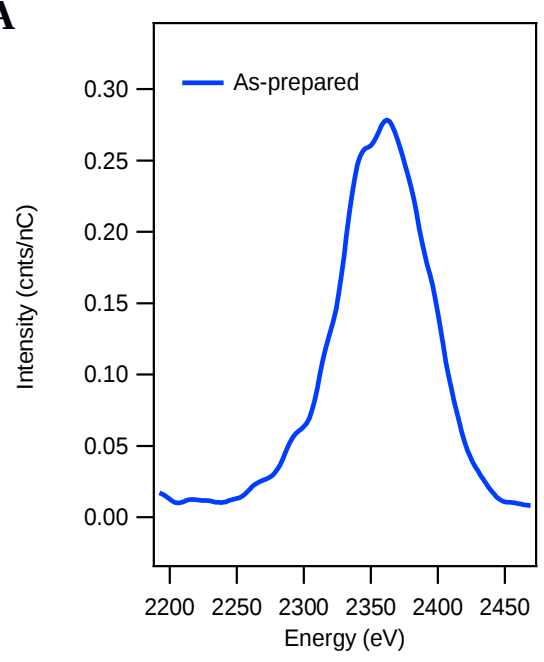

B

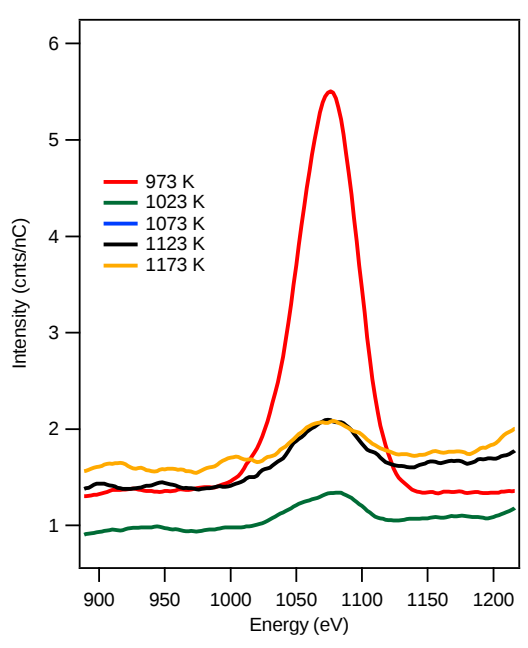

Figure S4. A) $\mathrm{He}^{+}$LEIS spectra of MAsA/Si(111) as-prepared in the As region; B) LEIS spectra from the oxygen region following high temperature annealing. We note that LEIS measurements are extremely surface sensitive and therefore strongly impacted by the cleanliness of the surface. The spectrum shown in Fig. $3 a$ in the main text was collected from a sample free of any hydrocarbon contamination; however, through the many experiments performed throughout the course of this work, we occasionally observed hydrocarbon contamination on the surfaces as a result of outgassing of the sample holder. This was observed for the case of the spectra shown in Fig. 3a of the main text (973 and $1023 \mathrm{~K}$ data points), resulting in an As surface peak with suppressed intensity relative to that observed here. This contamination, however, desorbed following the $1073 \mathrm{~K}$ anneal, so that the As LEIS spectra could be used for the LEIS quantification, as described next. 


\section{Supplemental Note 2: Quantitative LEIS analysis}

Quantification of LEIS data was performed using the TRBS code. ${ }^{9}$ The substrate was treated as semi-infinite $\mathrm{Si}$, with various types of $\mathrm{Si}_{x} \mathrm{As}_{\mathrm{y}}$ overlayers, as discussed below. A stopping power correction factor of 3.5 and a screening correction factor of 0.8 were applied. The calculations follow the trajectories of $10^{8} \mathrm{He}$ atoms incident on the sample with an initial energy of $3 \mathrm{keV}$. The trajectories were calculated until the energy falls below 500 $\mathrm{eV}$, and only the particles that scatter within an angular range of $144-146^{\circ}$ (corresponding to the acceptance of the Qtac analyzer) were included in the final spectra.

To evaluate the depth of the subsurface species as discussed in the main text, a multilayer system was constructed as a bulk Si substrate, followed by a $1 \mathrm{~nm}$ pure As layer, with a final pure Si overlayer of varying thickness (Fig. S7). The shift in the As binary collision peak due to the Si overlayer as compared to its position in the absence of a Si overlayer was matched to the shift in measured data to evaluate the correct depth.

Quantitative evaluation of the As concentration giving rise to this peak requires a priori knowledge of the reionization function of the Si(111) surface, as only ions are detected in LEIS measurements. A standard procedure has been established for such determination, ${ }^{8}$ and is performed as follows: first a measurement of a clean surface is obtained and the surface peaks are subtracted off. Second, a simulation of a bulk substrate is performed. The measurement is in effect the product of the simulation with the reionization function, so the reionization function is obtained by dividing the measured spectrum from the simulation (Fig. S8). With the knowledge of the reionization function, the results of the TRBS simulations can be scaled to enable direct comparison to measured data. The concentration of the subsurface As was evaluated by constructing a multilayer system comprised of Si $(6 \AA) / S i_{x} A s_{y}(10 \AA) / S i$, where the concentration $(x, y)$ is determined by trial and error. 


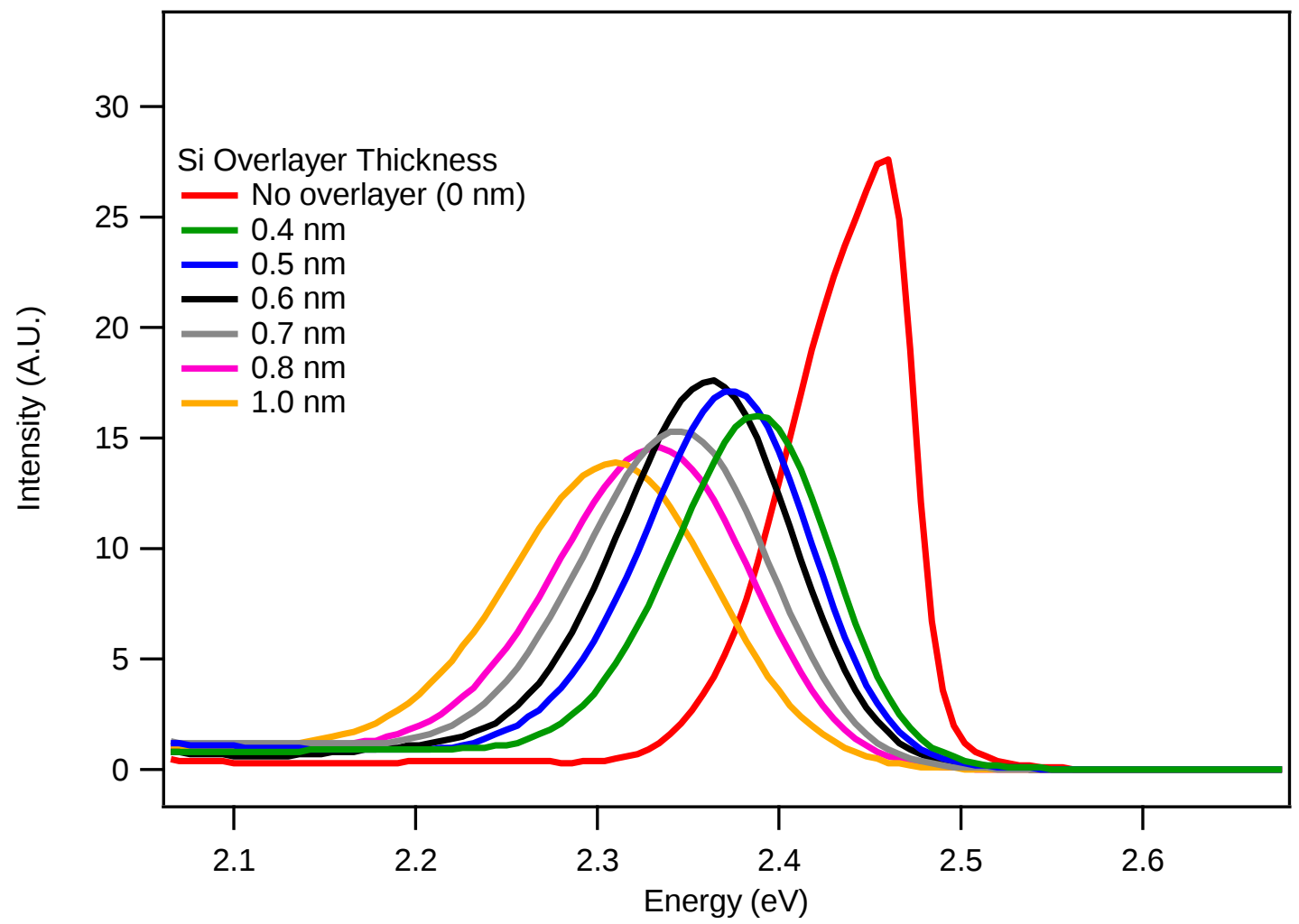

Figure S5. TRBS simulations of Si/As/Si multilayer structures. The calculations are performed by constructing multilayer structures comprised of a $1 \mathrm{~nm}$ As layer on a bulk Si substrate with varying thicknesses of an additional Si overlayer. In this manner, peak shifts in measured data can be correctly related to depth via the simulations. The calculations are performed via the TRBS code, ${ }^{9}$ assuming $10^{7}$ scattering events, with an electronic stopping correction factor of 3.5 and a screening correction factor of 0.8 . 

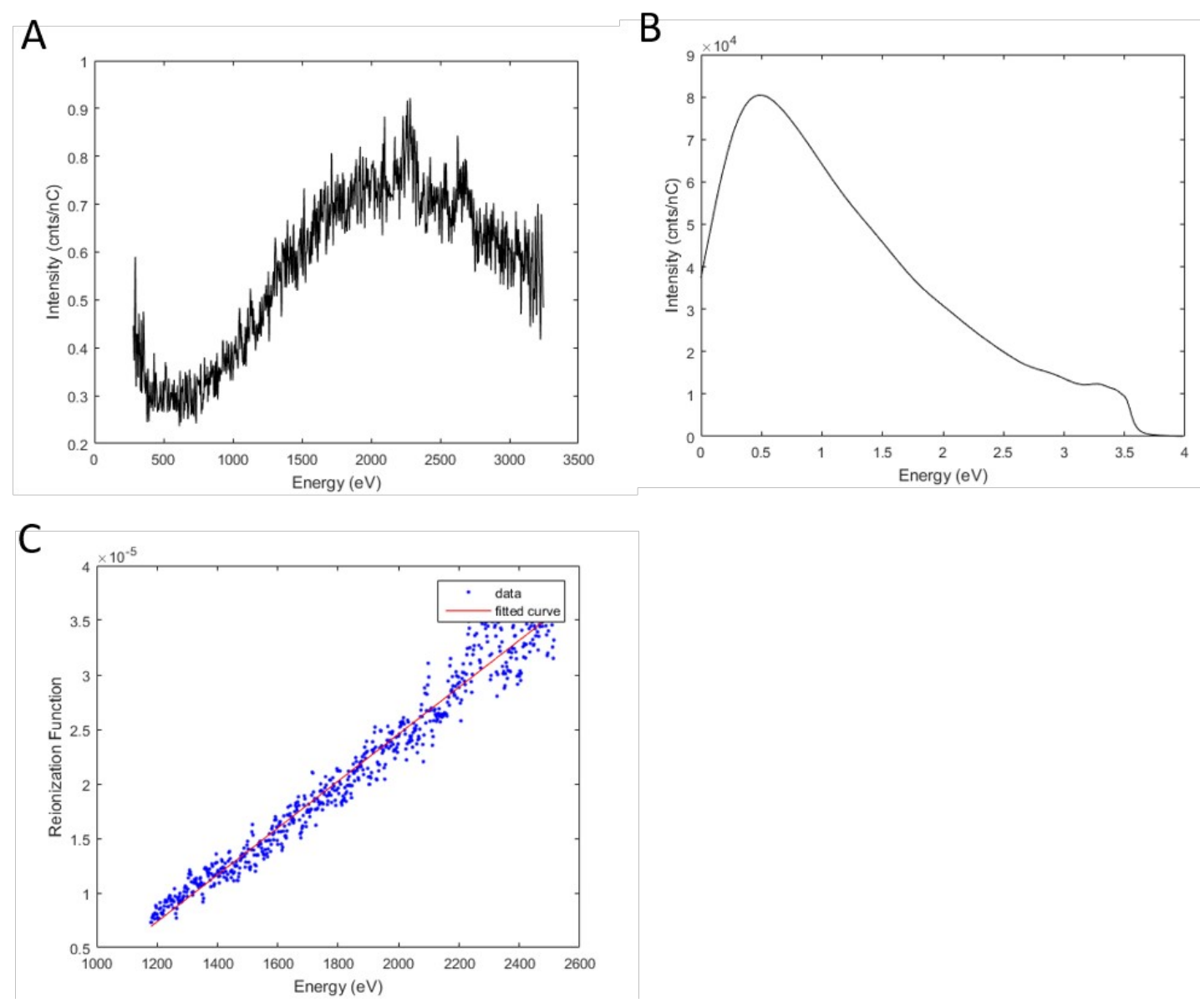

Figure S6. Determination of the reionization function for Si(111). A) $6 \mathrm{keV} \mathrm{He}+\mathrm{LEIS}$ spectrum taken from $\mathrm{Si}(111)$ upon annealing to $900 \mathrm{C}$, at which point all As is diffused below the surface. Additionally, using the higher ( $6 \mathrm{keV}$ ) ion energy, elastic peaks due to Si and As are shifted to higher energy, such that the As region from the $3 \mathrm{keV}$ data is unobscurred. B) TRBS simulations of a clean Si slab for $10^{8}$ dose of $6 \mathrm{keV} \mathrm{He}^{+}$. C) Extracted reionization function, obtained by dividing the measured data by the simulation, showing the expected linear ${ }^{8}$ behavior. 


\section{Supplemental Note 3: Quantitative Analysis of Broadband IR Response}

Analysis of the broadband IR spectra were performed using the RefFit ${ }^{10}$ optical fitting package. The system is treated as a $4 \mathrm{~nm}$ slab with uniform dielectric function, as the bulk of the substrate remains unchanged as a function of the annealing. The differential transmittance spectra taken following annealing are fitted using a differential dielectric function model. The model dielectric function includes two components; the first is a Drude function (centered at zero frequency) with plasma frequency of $\approx 2500$ $\mathrm{cm}^{-1}$ and broadening of $\approx 890 \mathrm{~cm}^{-1}$. The second is a Lorentzian peak centered at 4023 $\mathrm{cm}^{-1}$ with plasma frequency of $\approx 3900 \mathrm{~cm}^{-1}$ and broadening of $4883 \mathrm{~cm}^{-1}$. The change in the d.c. conductivity is obtained by extrapolating the fitted Drude component of the dielectric function to zero frequency. 

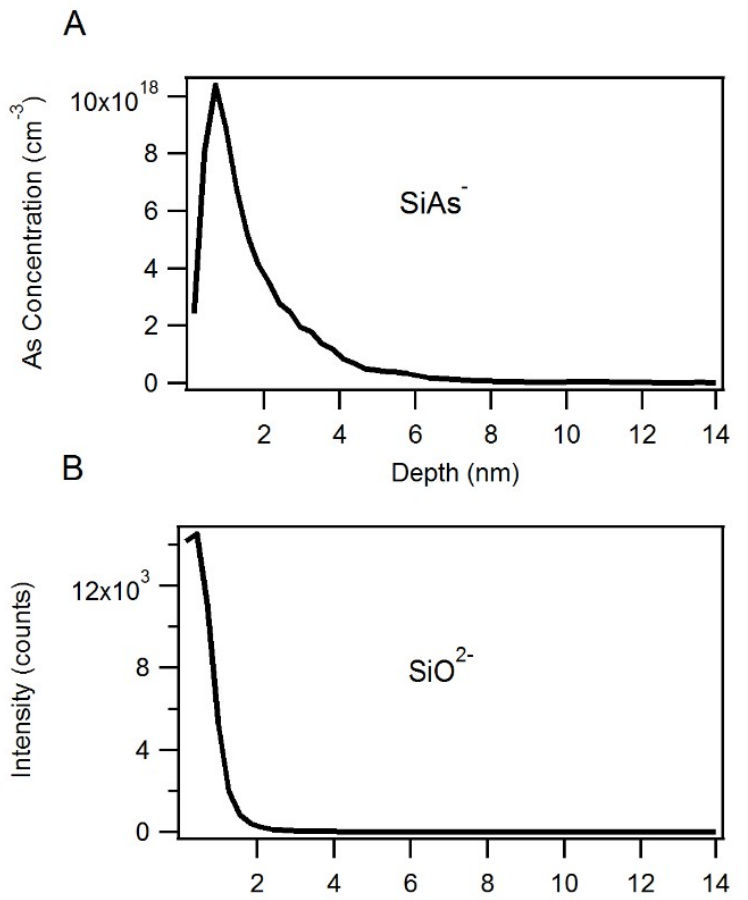

C

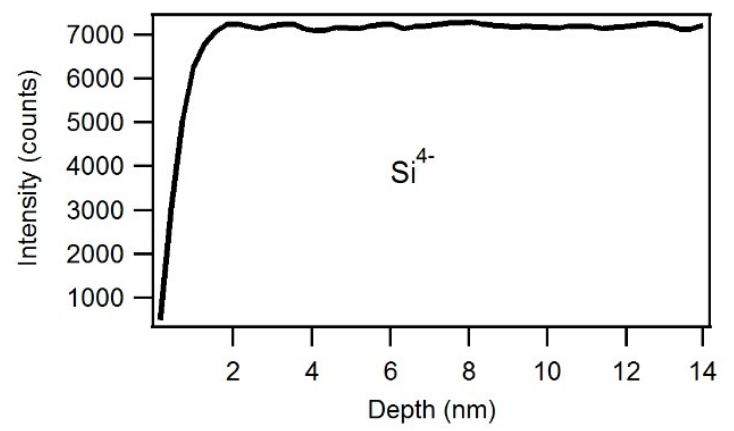


Figure S7. TOF-SIMS Measurements of MAsA monolayer-doped samples. A) Extracted concentration profile of As in the near surface region. Note that the concentration is determined from the SiAs- ion yield; however, the measurements are taken ex situ and the samples became heavily oxidized in the week between the sample preparation and SIMS measurements, as seen from the $\mathrm{SiO}^{2-}$ ion yield in (B). Consequently, the SiAs- ion yield is not representative of all of the As in the sample; rather, it is much more likely that As is bonded to Oxygen due to the high electron affinity of Oxygen. This is further illustrated by the Si4- ion yield in the near surface region (C), showing significant depletion of elemental $\mathrm{Si}$ in the near surface region. Thus, although the measurements do no provide an accurate absolute concentration of the As in the sample, the data do clearly demonstrate that As has diffused into the sample, and is peaked within $\approx 0.7 \mathrm{~nm}$ from the surface. Furthermore, the data underline the ultra-shallow nature of the MLD process. 


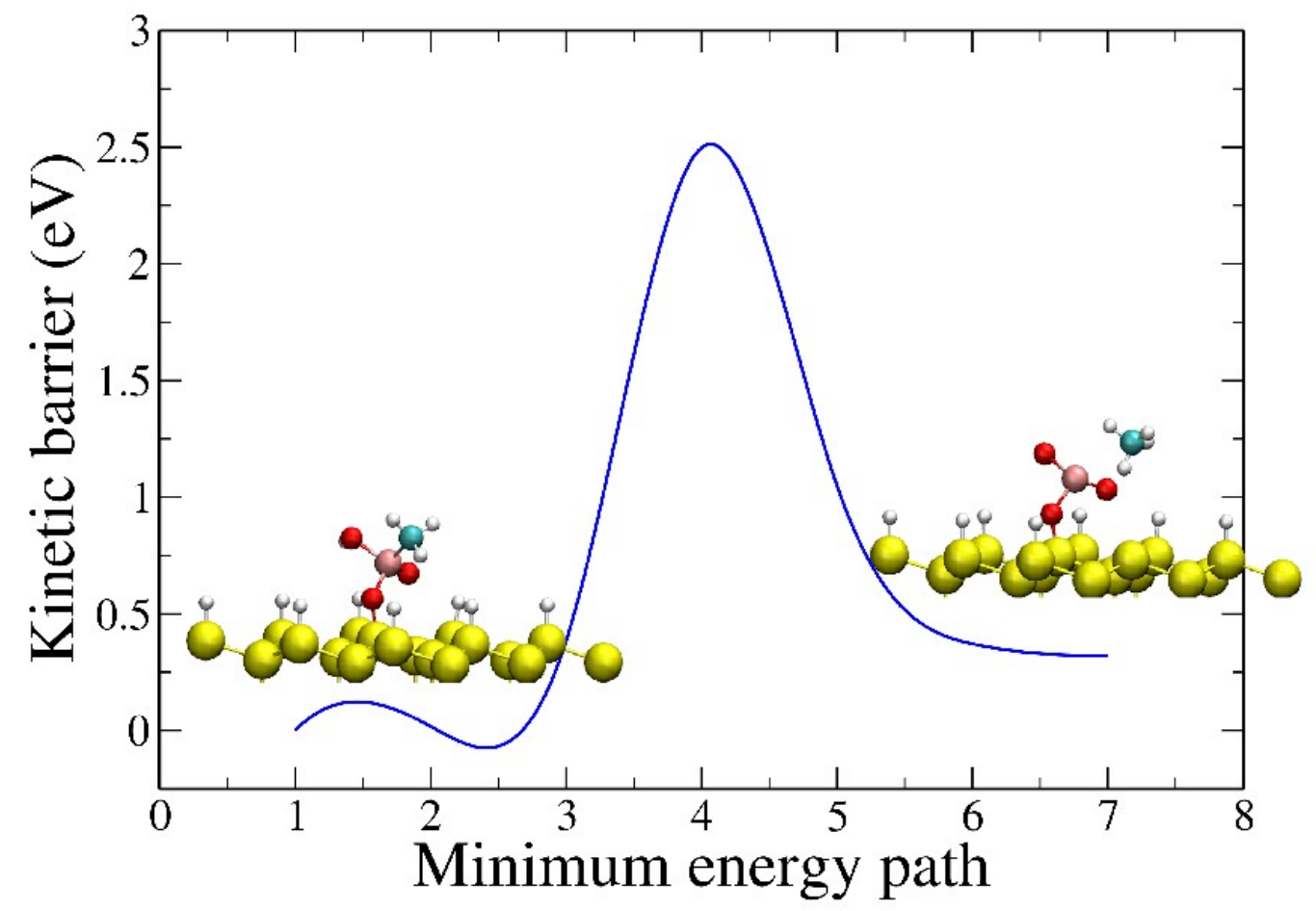

Figure S8. Kinetic barrier for the $\mathrm{CH}_{3}$ desorption from the adsorbed MAsA molecule. The insets show the initial and final states. Yellow spheres represent Si atoms; white, $\mathrm{H}$; blue, $\mathrm{C}$, red, $\mathrm{O}$ and pink spheres, As atoms, respectively. 


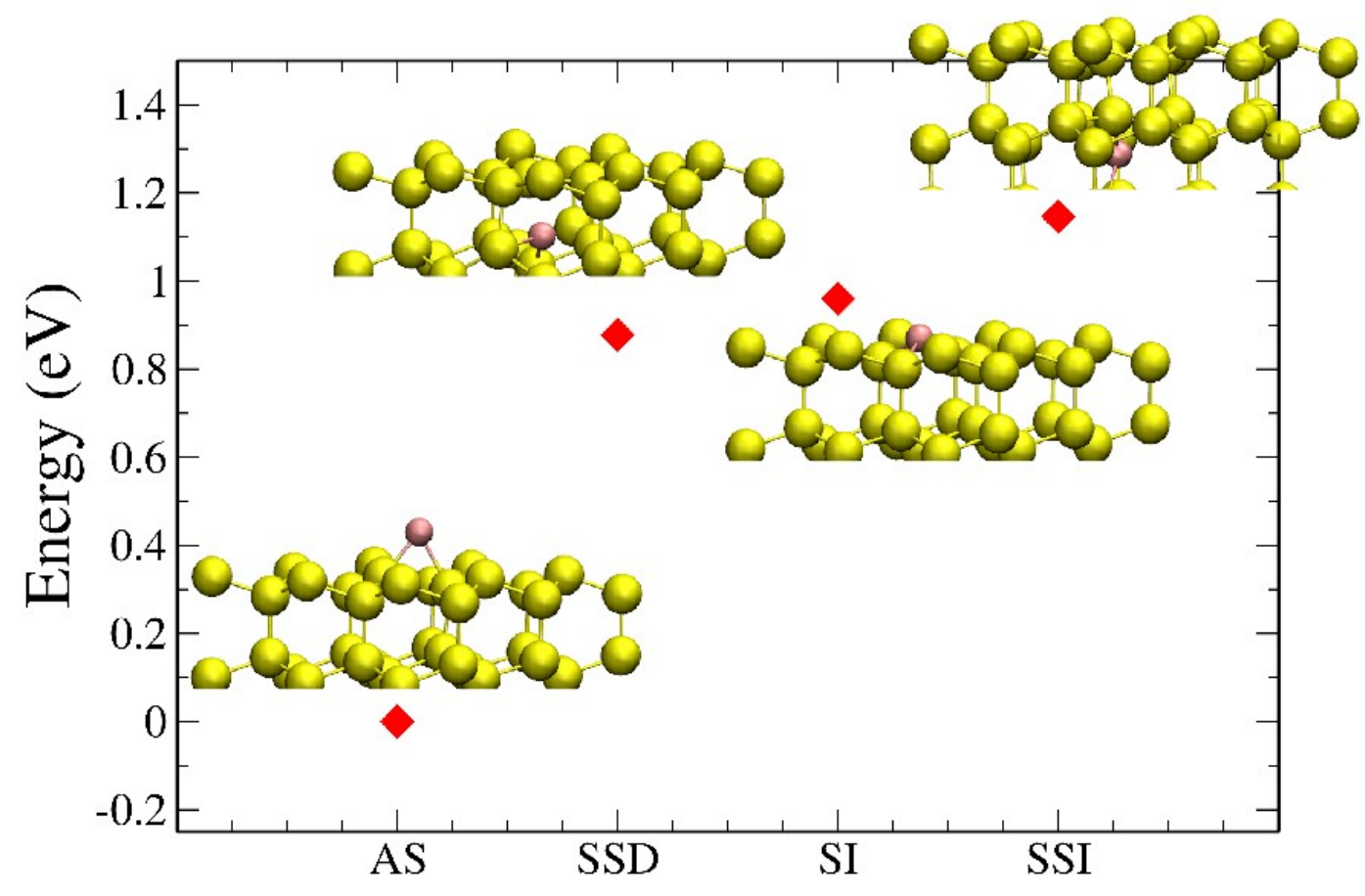

Figure 59. Relative energies for the different As doping configurations considered in this work. Adsorption on the surface (AS), sub-surface diffusion (SSD), surface interchange (SI) and sub-surface interchange (SSI). Yellow spheres represent Si atoms and pink spheres, As atoms, respectively. 


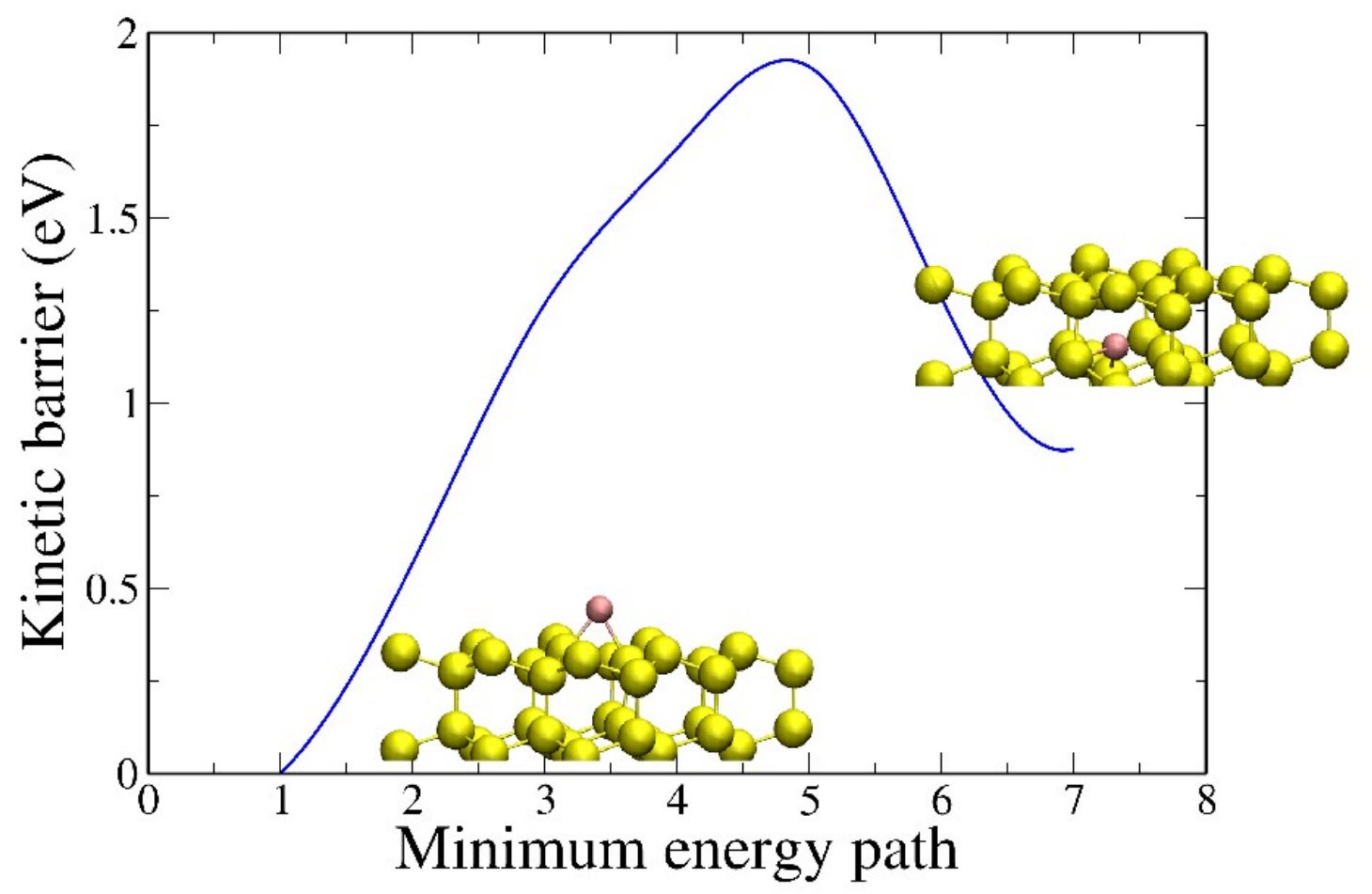

Figure S10. Kinetic barrier for As diffusion into the Si(111) surface (SSD configuration). The insets show the initial and final states. Yellow spheres represent Si atoms and pink spheres, As atoms, respectively. 


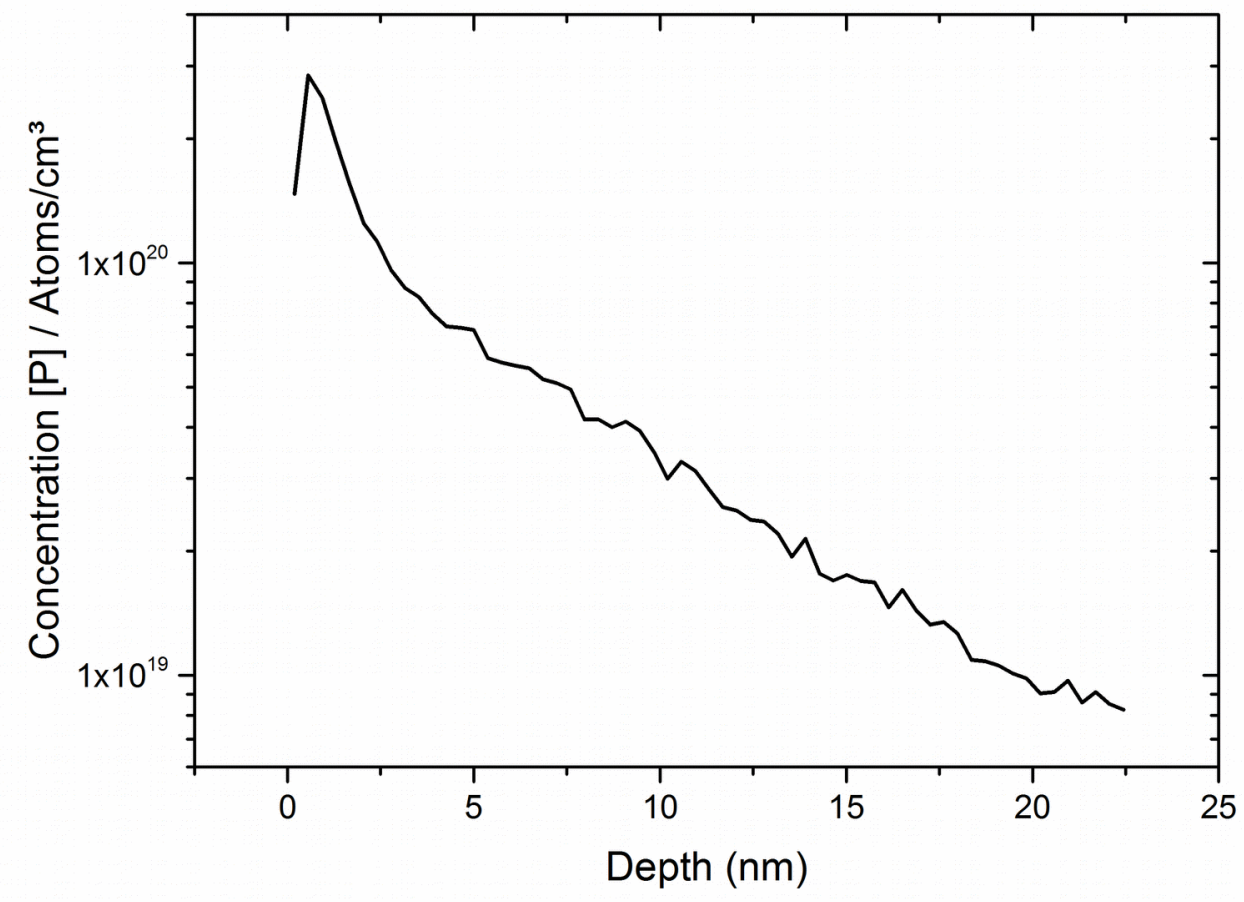

Figure S11. TOF-SIMS profiles of $\mathrm{P}$ in ODPA ML-Doped Si(111) obtained using the $\mathrm{Si}^{30 \mathrm{P}}$ isotope. One can observe a clear incorporation of phosphorus into the $\mathrm{Si}$ after heating the ODPA terminated sample. In this case, most of the P is detected with SIMS and the concentrations are closer to those extracted from LEIS for the As doping. 


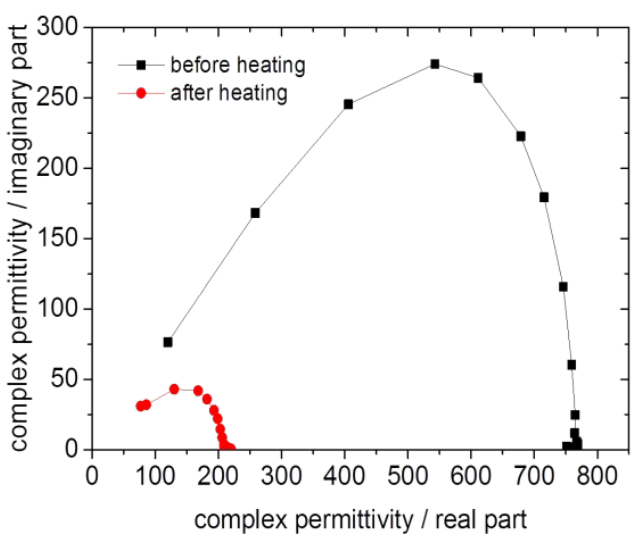

Figure S12. Electrical Characterization of ODPA ML-Doped Si(111). The real axis value at the low frequency intercept is the sum of the polarization resistance and the solution resistance. The change of the diameter of the semicircle is therefore equal to the change of the polarization resistance (in this case $-550 \Omega$ ) due to MLD. 


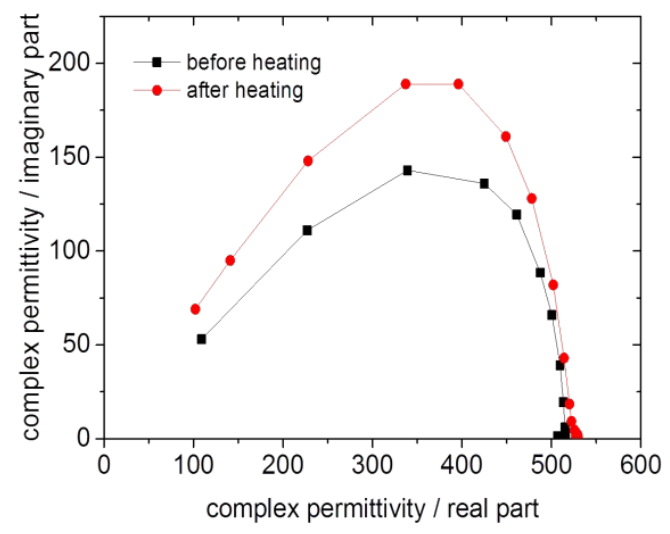

Figure S13. Electrical Characterization of clean hydrogen-terminated Si. The real axis value at the low frequency intercept is the sum of the polarization resistance and the solution resistance. The change of the diameter of the semicircle is therefore equal to the change of the polarization resistance (in this case negligible). 


\section{References}

1. Kresse, G.; Hafner, J. Ab Initio Molecular Dynamics for Liquid Metals. Phys. Rev. B 1993, 47, 558-561.

2. Kresse, G.; Furthmüller, J. Efficient Iterative Schemes for Ab Initio Total-Energy Calculations Using a Plane-Wave Basis Set. Phys. Rev. B 1996, 54, 11169-11186.

3. Kresse, G.; Joubert, D. From Ultrasoft Pseudopotentials to the Projector AugmentedWave Method. Phys. Rev. B 1999, 59, 1758-1775.

4. Monkhorst, H. K.; Pack, J. D. Special Points for Brillouin-Zone Integrations. Phys. Rev. B 1976, 13, 5188-5192.

5. Perdew, J. P.; Burke, K.; Ernzerhof, M. Generalized Gradient Approximation Made Simple. Phys. Rev. Lett. 1996, 77, 3865-3868.

6. Henkelman, G.; Uberuaga, B. P.; Jónsson, H. A Climbing Image Nudged Elastic Band Method for Finding Saddle Points and Minimum Energy Paths. J. Chem. Phys. 2000, $113,9901-9904$.

7. Henkelman, G.; Jónsson, H. Improved Tangent Estimate in the Nudged Elastic Band Method for Finding Minimum Energy Paths and Saddle Points. J. Chem. Phys. 2000, $113,9978-9985$.

8. Brüner, P.; Grehl, T.; Brongersma, H.; Detlefs, D.; Nolot, E.; Grampeix, H.; Steinbauer, E.; Bauer, P. Thin Film Analysis by Low-Energy Ion Scattering by Use of TRBS Simulations. J. Vacuum Science \& Technology A 2015, 33, 01 A122.

9. Biersack, J. P.; Steinbauer, E.; Bauer, P. A Particularly Fast TRIM Version for Ion Backscattering and High Energy Ion Implantation. Nuclear Instruments \& Methods in Physics Research Section B-Beam Interactions with Materials and Atoms 1991, 61, 7782. 
10. Kuzmenko, A. B. Kramers-Kronig Constrained Variational Analysis of Optical Spectra. Review of Scientific Instruments 2005, 76, 083108. 